\title{
Technology, Assembly and Test of a W-band Traveling Wave Tube for New 5G High Capacity Networks
}

\author{
Frédéric André, Member, IEEE, Jean-Clande Racamier, Ralph Zimmermann, Quang Trung Le, Viktor Krozer, \\ Giacomo Ulisse, Damien F. G. Minenna, Rosa Letizia, Senior Member, IEEE, and \\ Claudio Paoloni, Senior Member, IEEE
}

\begin{abstract}
The folded waveguide (FWG) traveling wave tube (TWT) developed in the frame of the Horizon 2020 TWEETHER project for enabling a novel W-band $(92-95 \mathrm{GHz})$ high capacity wireless network for 5G, is presented. The FWG TWT was designed by particle-in-cell simulations. The technology and the results in terms of measured $\mathrm{RF}$ losses and beam transmission from the realized beam tester are presented. Amplification on the first TWT breadboard has been observed but with a poor multi-reflection pattern resulting from spurious burrs inside the folded waveguide. It indicates however that the FWG technology offers great manufacturing simplification compared to conventional helix TWTs, thus enabling a low-cost device with large series production suitable for the wide market of wireless communications.
\end{abstract}

Index Terms-Traveling wave tube (TWT), Folded waveguide (FWG), W-band, wireless.

\section{INTRODUCTION}

W IRELESS communications at millimeter wave (30$300 \mathrm{GHz}$ ) are increasingly interesting to manufacturers and operators for multigigabit communications thanks to the availability of wide frequency bands. However, the high atmosphere attenuation at these higher frequencies hinders signal propagation over the required outdoor range. The low transmission power available from solid state devices above $60 \mathrm{GHz}$ [1], [2] forces the use of high gain antennas to enable links in the kilometer range. The new perspectives of $5 \mathrm{G}$ to provide an unprecedented capacity at terminal level and the request of very broadband fixed access pose substantial challenges in the new network architectures. High density small cell networks are envisaged as the solution for a capillary high coverage of the territory, especially in urban environment. Due to the high cost, low availability and lack of flexibility of the fibre, the wireless backhaul is considered the most promising solution to feed a multitude of base station in small areas. The distribution by Point to Multipoint (PmP), where a transmission hub feeds many small cell terminals would

This work is supported in part by the European Commission under the Horizon 2020 ICT-06-2014 grant number 644678 . (Corresponding author: Frédéric André)

D. F. G. Minenna PhD was supported by the Centre National d'Études Spatiales and Aix-Marseille University, CNRS, PIIM, UMR 7345

F. André, J-C. Racamier and D. F. G. Minenna are with Thales AVS/MIS, Vélizy-Villacoublay, 78140 France. (e-mail: frederic.andre@thalesgroup.com)

Q. T. Le and R. Zimmermann are with HFSE, Kassel, Germany.

G. Ulisse and V. Krozer are with Goethe University Frankfurth, Germany.

R. Letizia and C. Paoloni are with Lancaster University, UK. be cost effective and of easy deployment in comparison to the available Point to Point front ends. The PmP distribution needs a low gain antenna to provide a wide beam covering a sectorial area. Frequencies above $90 \mathrm{GHz}$ are still lightly regulated and low cost for operators. The W-band (92-95 $\mathrm{GHz}$ ) offers $3 \mathrm{GHz}$ bandwidth suitable for high capacity networks. The link calculation for PmP at W-band, to cover a sector with angle of about 90 degrees and $1 \mathrm{~km}$ radius, provided by a $16 \mathrm{dBi}$ antenna, results in a transmission power of about 40 Watt saturated. No solid state power amplifier can provide this level of power. Traveling Wave Tubes (TWTs) have been demonstrated to provide wide band high power amplification up to $1 \mathrm{THz}$, but so far their use is limited to space applications up to Q-band and defence where excellent results are obtained in W-band [3]. The European Commission Horizon 2020 Project TWEETHER developed the first Point to multiPoint system at W-band for high capacity wireless backhaul and access, based on a novel TWT [4], [5]. The enabling device is a folded waveguide (FWG) TWT designed to offer broadband and power efficient amplification at lower cost than conventional helix TWT to reach the broad market of wireless networks. The operating frequency band is 92 $95 \mathrm{GHz}$ (W-band) with $3 \mathrm{GHz}$ of instantaneous bandwidth for high data-rate. Two FWG TWTs were built and tested. This paper describes the design and fabrication challenges due to the small dimensions related to the operating frequency. Compared to previous work this project aims at bringing low power W-Band TWT at a higher maturity level in terms of cost, series production and application.

\section{Delay Line}

The FWG (see Fig. 1) delay line was chosen for this project because it is a broadband and simple structure which can be realized at low-cost. Its cross-section is $1.85 \times 0.3 \mathrm{~mm}^{2}$ with a pitch of $1.15 \mathrm{~mm}$ corresponding to an operating voltage of $16 \mathrm{kV}$. The beam tunnel is $0.5 \mathrm{~mm}$ in diameter.

In comparison to an equivalent helix (impractical at this frequency) it also allows a much larger beam tunnel. However, FWG are well known for their very low coupling impedance in comparison to the helix when operated away from cutoff for broadband operation. Typically the observed coupling impedance of FWG TWT designs is about 20 times weaker than that of helix TWT designs. The coupling impedance is 


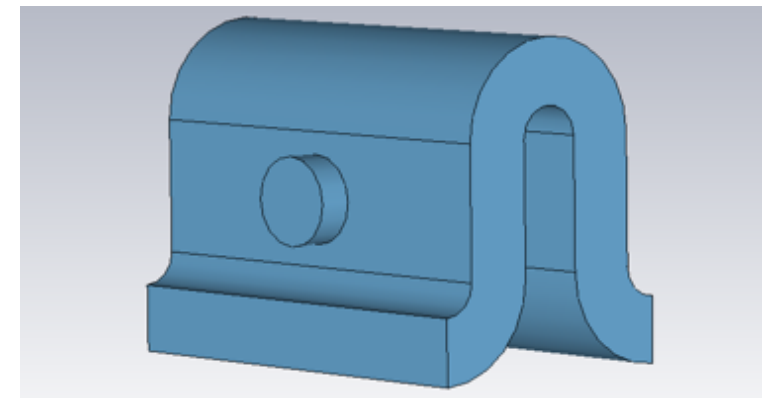

Fig. 1. Geometry of one longitudinal period of the FWG.

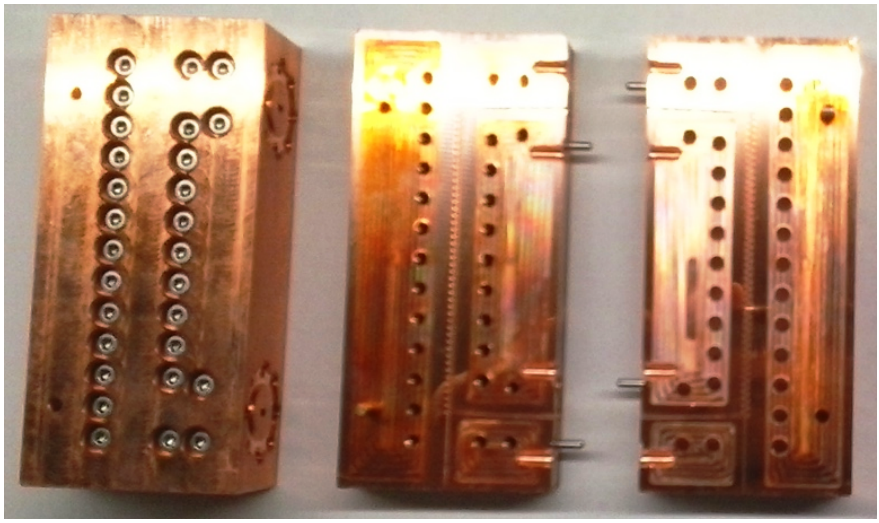

Fig. 2. Test FWG for loss and VSWR measurements. The vehicle is made of two symmetric halves screwed together.

the key parameter in a TWT because it quantifies how strong the electric field of the propagating wave is at the position of the electron beam. The stronger the coupling impedance the higher the beam efficiency (delivered RF power divided by the beam power). This beam efficiency deeply influences most TWT performances: its overall efficiency (for a given number of stages in the depressed collector), the cathode life time (through the required beam current), but also the RF losses along the amplification process. These losses are very important when the amplification process is very slow. Our numerical simulations show that in some realistic cases the RF power lost by skin effect on the FWG walls can be as high as the RF power at the output of the TWT. For this reason we carried out deeper analysis of the RF losses. The skin effect in our case depends on the conductivity and the surface roughness. We chose copper for its excellent conductivity and its machining easiness. The waveguide is made of two symmetric parts across its larger dimensions which need to be bonded for vacuum tightness. We assessed the losses in a test waveguide in copper with the TWT geometry (Fig. 2). Tapers to WR10 rectangular waveguide are used to access both extremities.

\section{FOLDED WAVEGUIDE FABRICATION AND TESTS}

An extensive experimental and simulation study on the behaviour of the FWG at W-band was conducted. FWG are realized by standard milling of pure copper. Two symmetric halves are realized, the contact surfaces between the two

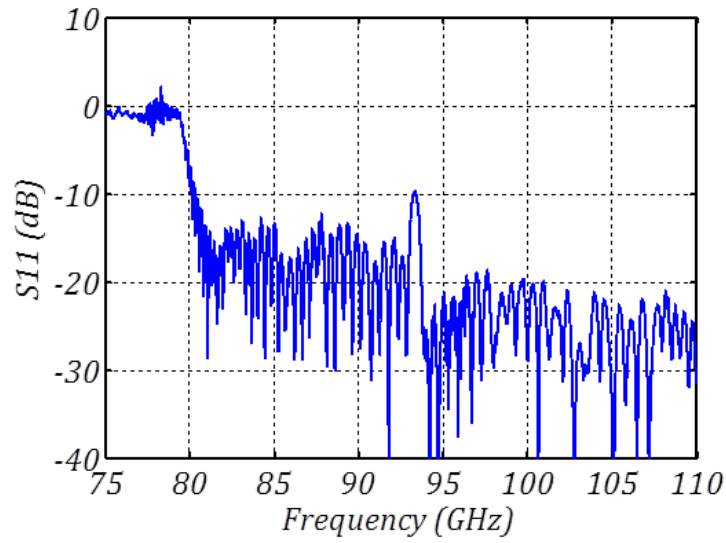

Fig. 3. Measured reflection coefficient $S_{11}$ of test waveguide showing rejection band peak.

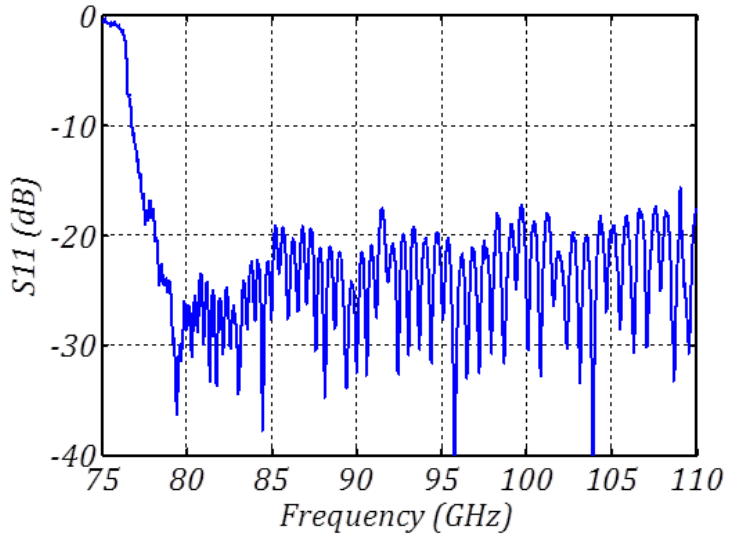

Fig. 4. $S_{11}$ of test waveguide with precise machining and alignment showing rejection band peak.

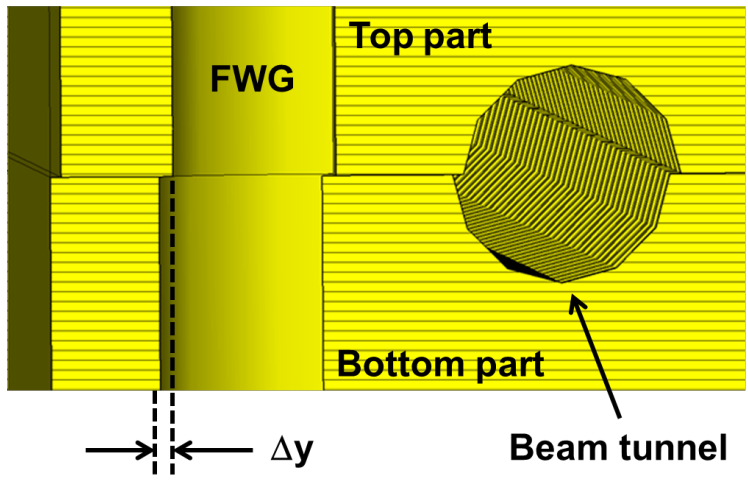

Fig. 5. Numerical simulation with an alignment shift $\Delta y$ of the two halves of the waveguide in the contact plan (cross-section of the geometry).

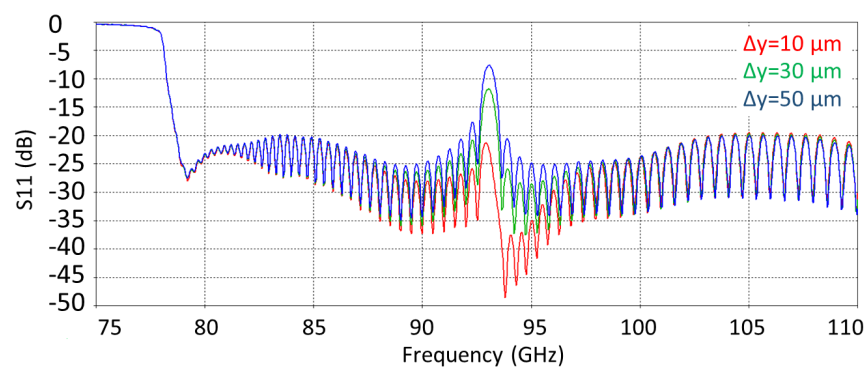

Fig. 6. Measured coefficient of reflection in the simulation for different values of the $\Delta y$ shift. 
Fig. 7. Two stages FWG TWT.

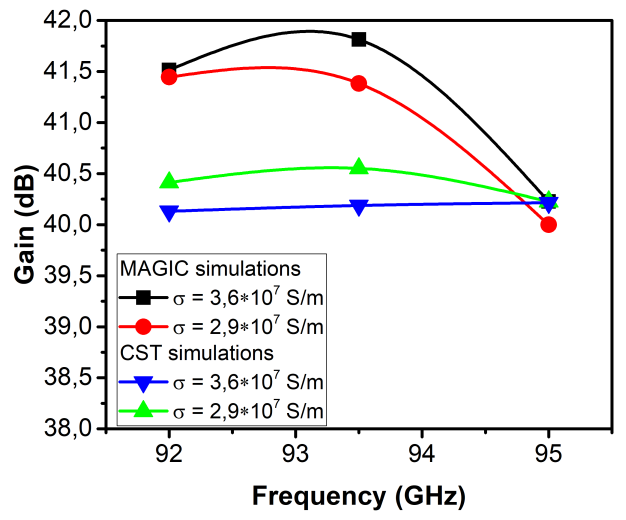

Fig. 8. Gain simulated by MAGIC3D and CST-PS with two different equivalent conductivities $\sigma$ for the copper walls of the FWG.

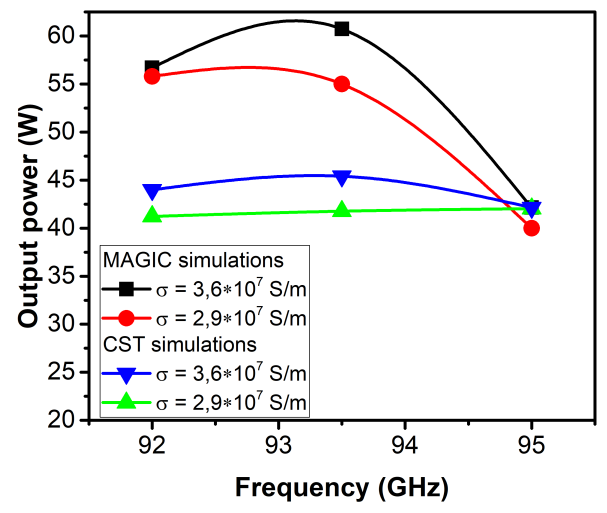

Fig. 9. Power simulated by MAGIC3D and CST-PS with two different equivalent conductivities $\sigma$ for the copper walls of the FWG.

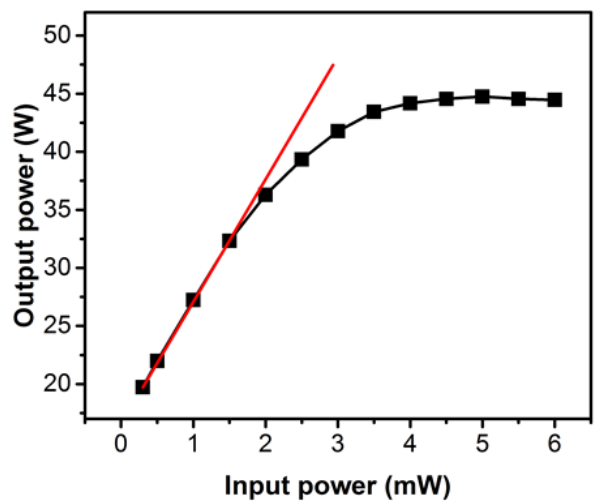

Fig. 10. Output power versus drive power (the straight line indicates the linear gain).

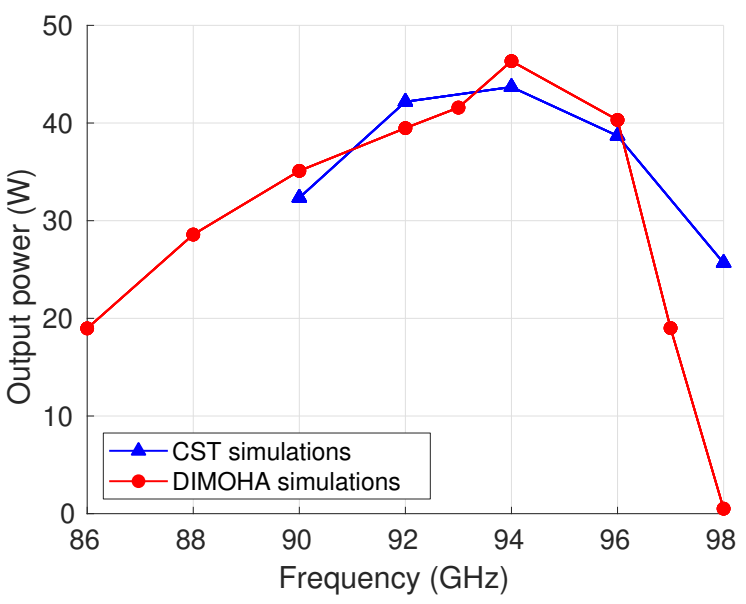

Fig. 11. Saturated output power versus input power simulated with CST-PS (blue triangles) from Ref. [6] and with DIMOHA (red dots).
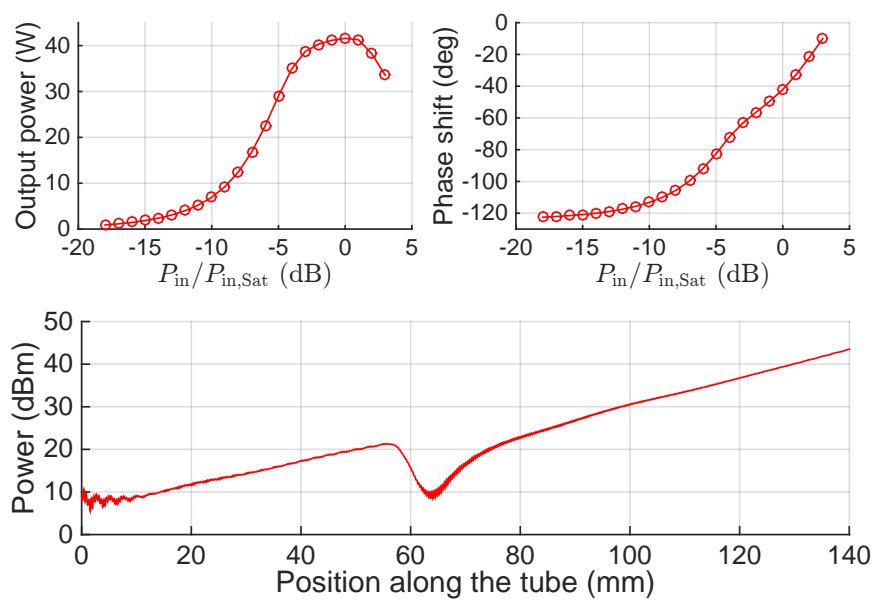

Fig. 12. Simulations of output power and phase shift versus input back-off at $93 \mathrm{GHz}$ with DIMOHA (top) and output power along the axis (bottom, small power oscillation near input is an artifact of the injection method, smooth progressive attenuation in the middle is an approximation of the actual localized sever)

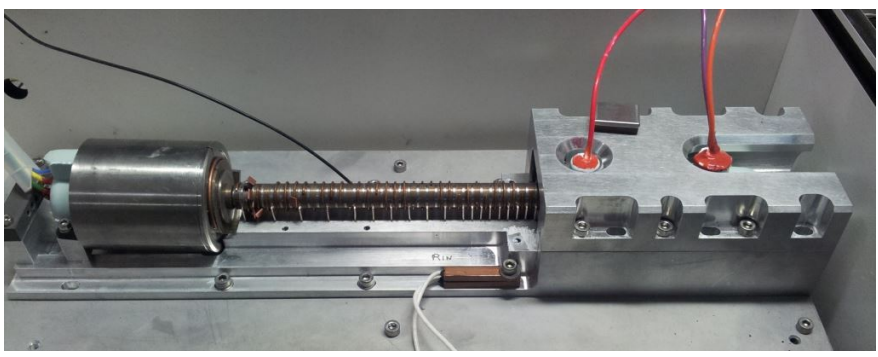

Fig. 13. Beam tester under operation. 


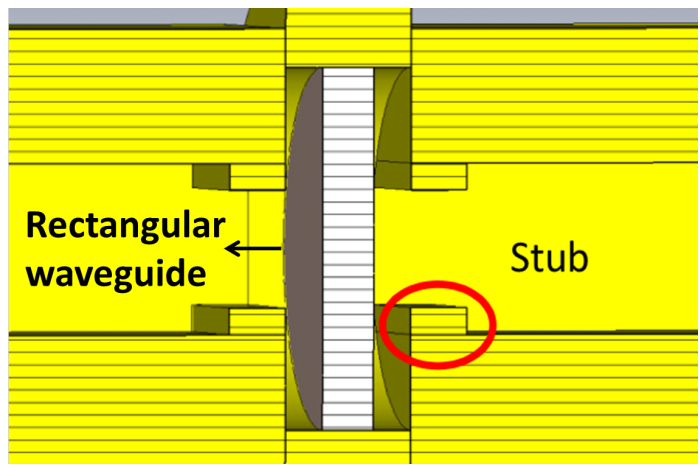

Fig. 14. RF Window schematic cross-section (metal in yellow, center ceramic disc in white)

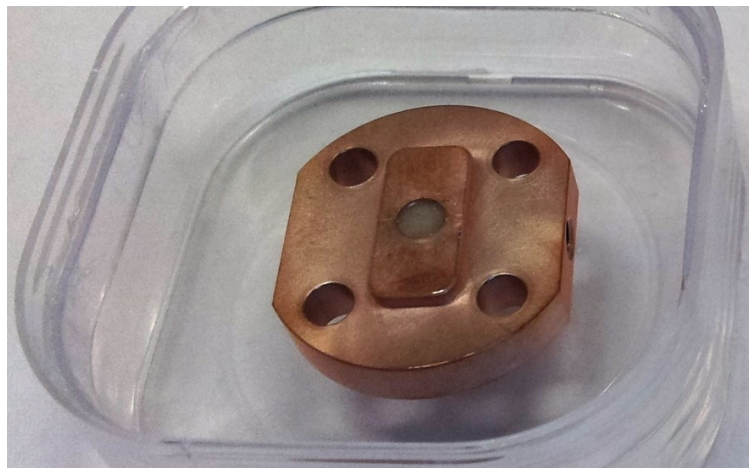

Fig. 15. Brazed RF window.

halves are prepared with low roughness $\left(R_{a}<200 \mathrm{~nm}\right)$ and high flatness $< \pm 5 \mu \mathrm{m}$ across the surface.

\section{A. Losses measurement}

Losses are an important parameter for evaluating RF output power and the length of the FWG. We need to provide the simulation with a reliable losses value. Two techniques are used for loss measurements. In the first one we measure the transmission losses $S_{21}$ through the test waveguide and we divide by the FWG length to find $47 \mathrm{~dB} / \mathrm{m}$ of losses. This technique assumes that the two access section have negligible losses. In order to de-embed the FWG from the two access ports we have placed a movable short circuit inside the beam tunnel. The amplitude and position of the short circuit are determined by time domain analysis (Fourier transform) with the vector network analyser on the reflection parameter $S_{11}$. When the perturbation moves away from the analyser its amplitude is fading, thus giving the losses as a function of the position (this bead pull technique was previously described in [7]). This second technique gives a value very close from the previous one, $45.5 \mathrm{~dB} / \mathrm{m}$, and slightly below as expected. The losses can be expressed in terms of equivalent conductivity, i.e. the wall conductivity giving the same loss for an ideal waveguide without roughness. We find a value of $2.7 \times 10^{7} \mathrm{~S} / \mathrm{m}$ which is similar to the value found by other teams working on similar devices. For example, Hu et al. [8] found $2.25 \times 10^{7} \mathrm{~S} / \mathrm{m}$. The deviation from the conductivity of pure copper $\left(5 \times 10^{7} \mathrm{~S} / \mathrm{m}\right)$ is likely resulting from surface roughness. The literature shows that the ideal conductivity is reached with $20 \mathrm{~nm}$ roughness, which is feasible by standard milling with diamond cutters [9], but is probably not achieved in our project. A first measurement at the bottom of the groove gives $\mathrm{Ra}=320 \mathrm{~nm}$.

\section{B. Reflection coefficient measurement}

A number of FWGs were built for measuring the reflection coefficient $S_{11}$ with the tapered transitions. Some broadband measurements (Fig. 3) exhibit a reflection resonance at $94 \mathrm{GHz}$. This phenomenon is well explained by a breaking in the FWG symmetry due to misaligned machining or assembly [10]. This is confirmed in our numerical simulations with different misalignments replicating real structures (Fig. 5 and 6). Careful machining and alignment of the two halves succeed in reducing this resonance at very low level in subsequent FWG realisation (Fig. 4). Our experience is that this kind of resonance can lead to a loss of power in the corresponding frequency range (power holes) and, in some cases, to spurious oscillation. For this reason we made the decision to slightly reduce the larger side of the FWG in order to shift the resonance slightly above the operating band. Our CST-PS PIC simulations showed that the design is free from oscillation in the presence of the resonance. Finally it is noticeable that the return losses are around a satisfactory $20 \mathrm{~dB}$ over a very broad band.

\section{TWT DESIGN AND PERFORMANCE}

The FWG TWT design specifications are $40 \mathrm{~W}$ output power at saturation and $40 \mathrm{~dB}$ gain. As depicted in Fig. 7 the simulated delay-line consists of two cascaded sections of FWG with severs in-between (not represented), the usual architecture for this kind of device. Severs are RF loads used to attenuate the signal. They are usually made of carbonized ceramic [8] or complex lossy ceramic based on Silicon carbide. In our case they are made of graphite for easier machining and reduced cost. A simulation campaign using two different PIC simulators, CST-PS and MAGIC3D, was conducted. Two different levels of copper losses were considered to better evaluate impact of losses on performances. The full TWT was simulated assuming the beam confined by a magnetic focussing field of 0.3 Tesla. Both simulators predict a gain larger that $40 \mathrm{~dB}$ and an output power larger than $40 \mathrm{~W}$. However, the difference in output power (60 W in MAGIC3D compared to $45 \mathrm{~W}$ ) is not negligible, due to the different models used for the mesh and the electron interaction. Fig. 8 and Fig. 9 show the gain obtained by the two simulators. The behaviour for $\sigma=3.6 \times 10^{7} \mathrm{~S} / \mathrm{m}$ is quite similar with a difference lower than $1.5 \mathrm{~dB}$ for the gain. Fig. 9 shows the output power computed by MAGIC3D for the two different values of copper conductivity. Fig. 10 shows the Pout vs. Pin curve computed by CST-PS. We also checked the stability of the TWT with CST, no oscillations have been detected.

To differenciate between CST-PS and MAGIC3D, a second simulation campaign was performed this time using our new Discrete Model with Hamiltonian approach DIMOHA [11], a specialized 1D time-domain code based on a many-body description [12]. It is well benchmarked against helix TWTs 
but also works on FWG TWTs. DIMOHA is greatly faster ( $2 \mathrm{~h}$ for $6 \mathrm{~ns}$ on a laptop with 4 processors) thus permitting to calculate accurate AM/AM and AM/PM curves. For this simulation only the first mode of propagation was taken into account as it is known that higher modes can be neglected. Figure 11 shows the output power at saturation versus frequency. Large passband is predicted (86-97 GHz), in agreement with preliminary measurement. We estimate discrepancies at 98 $\mathrm{GHz}$ between CST-PS and DIMOHA may occur because we have no reliable dispersion relation above this frequency only extrapolation for second harmonics. In Fig. 12, the output power and the phase shift versus the input power are plotted for $F=93 \mathrm{GHz}$.

\section{BEAM TESTER}

The success of the project is related to a high quality and well confined electron beam. Once defined the beam parameters, $16 \mathrm{kV}$ beam voltage and $70 \mathrm{~mA}$ beam current, a beam tester was designed and realized. This is a device consisting in a complete TWT where the delay-line is replaced by a copper pipe with a tunnel diameter slightly smaller $(0.4 \mathrm{~mm})$ and the same length as those of the final design. All properties of electron optics and cathode emission can be tested exactly like the final TWT in no-drive. The electron gun is a standard Pierce gun adapted from a telecommunication helix tube with a theoretical beam filling factor of $50 \%$. The targeted beam current, $70 \mathrm{~mA}$ in space charge regime, corresponds to an expected cathode life-time of 5 years based on our experience with M-type cathode. This life-time is quite sensitive to the beam current (e.g. 15 years at $45 \mathrm{~mA}$ and 10 years at $53 \mathrm{~mA}$ ). Higher temperature is required to emit more current due to the Richardson-Duschman law of emission, with a negative impact on the life-time. Reducing the beam current to $50 \mathrm{~mA}$ for example doubles the life-time. It is expected to operate the TWT with lower current because the initial design with $70 \mathrm{~mA}$ is based on conservative hypothesis on losses and bandwidth, as illustrated by the simulation. Furthermore, bandwidth can be reduced by operating closer to the cut-off of the waveguide where the coupling impedance is larger, to the benefit of the beam efficiency. So our bandwidth margin can be traded against lower beam current and longer life-time.

We are using the two stage collector and the PPM (periodic permanent magnet) system from other telecom tubes. They are tested as well in the beam tester (Fig. 13). This device validates many design options on electron optics, magnetic field, collector and several assembling process. A beam transmission better than $95 \%$ is obtained on the first device with stable behaviour over more than 3000 hours of $\mathrm{CW}$ operation when this test was intentionally stopped.

\section{RF WINDOW}

$\mathrm{RF}$ windows are needed to propagate the RF signal inside and outside the slow wave structure and to ensure vacuum tightness. The VSWR of the window as well as its losses need to be low in a broad band around the operating frequency. The first designed window was based on a disk of alumina in the "pill-box" configuration. Even though the first design was

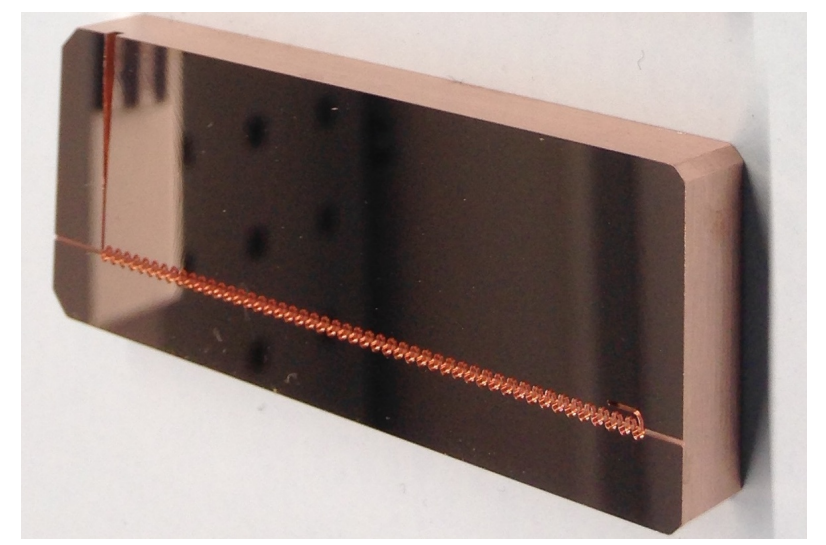

Fig. 16. Final FWG in copper for the TWT: half part prior to diffusion bonding. Machining to the final outer dimensions is done after bonding.

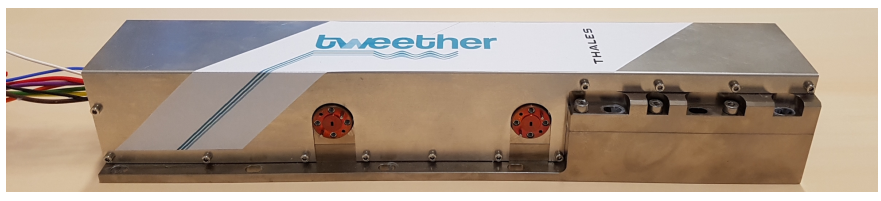

Fig. 17. Assembled W-band TWT A. Length is $345 \mathrm{~mm}$, width $72 \mathrm{~mm}$ and height $54 \mathrm{~mm}$.

satisfactory, an optimization was performed to further reduce the VSWR and to increase the bandwidth. Further, a thinner window is used in order to reduce losses. With respect to the first window design four stubs were introduced as shown in Fig. 14. The stubs are used to optimize the transition between the rectangular and the circular waveguide. The $S_{11}$ parameters is below $-20 \mathrm{~dB}$ over a large bandwidth. Several dielectric constants were simulated since it could slightly change with the actual alumina. In all cases the $S_{11}$ remains below $-20 \mathrm{~dB}$. Realized RF windows are shown in Fig. 15 after the ceramic brazing step.

\section{FINAL ASSEMbly}

Two TWTs were built (named TWT A and B). The two halves of the FWG have to be bonded to have a perfect vacuum tightness. The usual bonding process is brazing with gold or silver. However, brazing is always accompanied by diffusion of the brazing material resulting in the formation of gold or silver alloy on the walls of the FWG. Even a small amount of gold into copper causes strong reduction of its conductivity [13]. For this reason we use a diffusion bonding process to preserve pure copper conductivity. A good surface finish is required at the interface of the to part as shown in Fig. 16. The main difficulty during TWT assembling was to obtain good vacuum tightness after the diffusion bonding process. The tightness is controlled by conventional helium test. We encountered vacuum tightness problems which were solved by complementing the initial diffusion bonding step by a standard brazing step. The photo of the first W-band FWG TWT is shown in Fig. 17.

After assembling the RF performances were measured. The beam current is $25 \mathrm{~mA}$ and the collector voltages 4.25 and $1.35 \mathrm{kV}$ wrt cathode. The beam transmission is only $80 \%$ 
due to waveguide deformations which are shown hereunder. Figure 18 shows the measurements of the gain for TWT A with $-15 \mathrm{dBm}$ input power. Only few peaks at $20 \mathrm{~dB}$ are observed instead of the predicted flat $40 \mathrm{~dB}$ gain from simulations. Using X-ray tomography, we identified two causes. As show in Fig. 19 (left part), the waveguide walls of TWT A are deformed. There is also a small misalignment $\Delta y$ between the two FWG parts (no visible in Fig. 19). Most importantly, there are small unwanted particles inside the folded waveguide. The manufacturing of the FWG was clearly improved for the second TWT (B). As show in Fig. 19 (right part), there is no wall deformation nor misaligment. Hovewer, unwanted particles still forbid wave propagation, therefore, flat amplification is still not observed. Methods to remove those particles are under investigation.

Finally, it should be noted that the delay line based on this FWG is much simpler than a conventional helix system in terms of piece part numbers and associated processes (like welding, attenuation...). The main simplification result from the elimination of helix supporting rods and transition to the output waveguide. The complex connection of the helix to the output waveguide with matching element pertaining to the helix structure is drastically simplified with a simple unfolding at the end of the FWG implemented inside the FWG part itself. We succeeded to design a FWG delay line using only 3 pieces (two FWG parts and sever) and 3 assembling processes (diffusion bonding and final brazing steps). This simplicity has a large impact on the manufacturing cost of the complete TWT and will participate to large volume production at low cost.

\section{CONCLUSION}

The design and fabrication of a FWG TWT to enable the first point to multipoint W-band wireless networks has been described. The expected performance have been simulated. A beam tester has successfully demonstrated the high electron beam quality. The targeted flat amplification was not achieved on both manufactured TWTs, only resonant peaks of amplification were observed with a multi-reflection pattern. The reason is clearly the presence of parasitic particles and deformation inside the waveguide. Part of these problems have already been solved on the second TWT, but still requires a few additional optimization iterations after this project. We have now a reproducible process that allows building and testing of the TWTs. Finally, a substantial reduction in fabrication complexity has been demonstrated at design level for affordable TWT production.

\section{REFERENCES}

[1] J. M. Schellenberg, "A 2-W W-Band GaN Traveling-Wave Amplifier With 25-GHz Bandwidth," IEEE Trans. Microw. Theory Techn., vol. 63, no. 9, Sep. 2015, pp. 2833-2840. doi: 10.1109/TMTT.2015.2453156.

[2] A. Margomenos, A. Kurdoghlian, M. Micovic, K. Shinohara, D. F. Brown, A. L. Corrion, H. P. Moyer, S. Burnham, D. C. Regan, R. M. Grabar, C. McGuire, M. D. Wetzel, R. Bowen, P. S. Chen, H. Y. Tai, A. Schmitz, H. Fung, A. Fung, D. H. Chow, "GaN Technology for E, W and GBand Applications" in IEEE Compound Semiconductor Integrated Circuit Symposium (CSICS), La Jolla, CA, USA, Oct. 2014, pp. 1-4. doi: 10.1109/CSICS.2014.6978559.

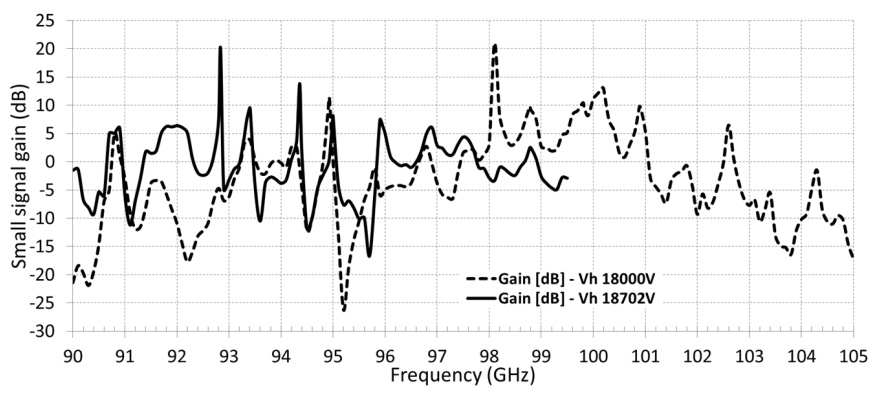

Fig. 18. Measured gain of TWT A for two cathode potentials $V_{h}$.

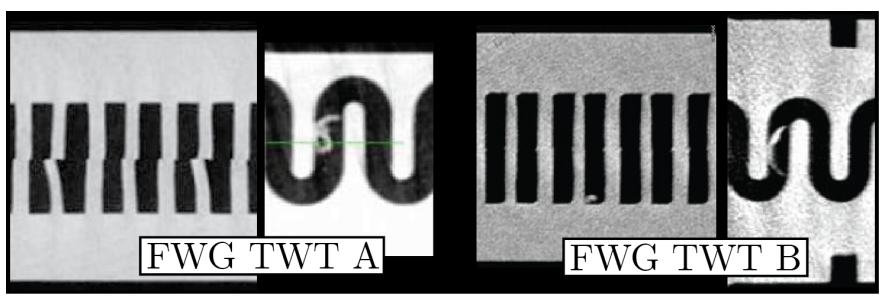

Fig. 19. X-ray tomography of the FWG of TWT A and B showing deformation and parasitic particles.

[3] R. Kowalczyk, A. Zubyk, C. Meadows, M. Martin, M. Kirshner, R. True, A. Theiss, J. Rominger and C. Armstrong, "A 100 Watt W-Band MPM TWT," 2013 IEEE 14th International Vacuum Electronics Conference (IVEC), Paris, 2013, pp. 1-2. doi: 10.1109/IVEC.2013.6571123

[4] C. Paoloni, F. Magne, F. André, X. Begaud, V. Krozer, M. Marilier, A. Ramirez, R. Vilar and R. Zimmerman, R 2017, "TWEETHER future generation W-band backhaul and access network technology" in European Conf. Networks Comm. (EuCNC), Oulu, Finland, Jun. 2017, pp. 1-5. doi: 10.1109/EuCNC.2017.7980684.

[5] TWEETHER website [Online]. Available: http://www.tweether.eu.

[6] Andre, F, Kohler, S, Krozer, V, Quang, TL, Letizia, R, Paoloni, C, Sabaawi, A, Ulisse and G \& Zimmerman, R 2017, "Fabrication of W-band TWT for 5G small cells backhaul" in Proc. 18th Int. Vac. Electron. Conf. (IVEC), London, U.K., Apr. 2017, pp. 1-4. doi: 10.1109/IVEC.2017.8289653.

[7] H. Büssing, A. Grede and H. Henke, "First Measurement of a W-Band Folded Waveguide RF Structure," 2014 IEEE Int. Vacuum Electronics Conference. doi: 10.1109/IVEC.2014.6857551

[8] Y. Hu, J. Feng, J. Cai, X. Wu, Y. Du, J. Liu, J. Chen, and X. Zhang, "Design and experimental study of a widebandwidth W-band folded waveguide continuous-wave TWT," IEEE Trans. Plasma Sci., vol. 42, no. 10 , Oct. 2014, pp. 3380-3386. doi: 10.1109/TPS.2014.2350477.

[9] I. Stil, A. Fontana, B. Lefranc, A. Navarrini, P. Serres, and K. Schuster, "Loss of WR10 waveguide across 70-116 GHz" in 23rd Int. Symp. Space Terahertz Tech., Tokyo, Japan, Apr. 2012, pp. 151-153, 2012.

[10] K. T. Nguyen, A. N. Vlasov, L. Ludeking, C. D. Joye, A. M. Cook, J. P. Calame, J. A. Pasour, D. E. Pershing, E. L. Wright, S. J. Cooke, B. Levush, D. K. Abe, D. P. Chernin, and I. A. Chernyavskiy, "Design methodology and experimental verification of serpentine/foldedwaveguide twts," IEEE Trans. Electron Devices, vol. 61, no. 6, Jun 2014, pp. 1679-1686. doi: 10.1109/TED.2014.2303711.

[11] D. F. G. Minenna, Y. Elskens, F. André, J. Puech, A. Poyé and F. Doveil, "DIMOHA: A Time-Domain Algorithm for Traveling-Wave Tube Simulations," IEEE Trans. Electron Devices, vol. 66, no. 9, Sep. 2019, pp. 4042-4047. doi: 10.1109/TED.2019.2928450.

[12] D. F. G. Minenna, Y. Elskens, F. André and F. Doveil, "Electromagnetic power and momentum in N-body Hamiltonian approach to wave-particle dynamics in a periodic structure," Europhys. Lett., vol. 122, no. 4, Jul. 2018, Art. no. 44002. doi: 10.1209/0295-5075/122/44002.

[13] M. W. Ackerman, K. Y. Wu, T. N. Havill, R. H. Bogaard, R. A. Matula, S. G. Oh, and H. M. James, "Electrical resistivity of ten selected binary alloy systems," J. Phys. Chem. Ref. Data, vol. 12, no. 2, Oct. 1983, pp. 183-322. doi: 10.1063/1.555684. 